\title{
El Cambio Climático en la ESO. Una aproximación exploratoria en Extremadura
}

\author{
The climate change in the ESO. Exploring Extremadura case
}

Manuela Caballero Guisado y Artemio Baigorri Agoiz

\section{Resumen}

Desde que en 1988 la Asamblea General de la ONU aprobase el Panel Intergubernamental sobre Cambio Climático (IPCC) han sido cinco los informes, el último publicado a finales de 2014, que alertan de las consecuencias que la elevación de la temperatura del planeta puede tener para los ecosistemas que mantienen la vida en la tierra, y por tanto para las sociedades humanas. El informe recomienda a los decisores políticos que tomen medidas que faciliten la capacidad de resiliencia y adaptación de los ciudadanos ante ese Cambio Climático (en adelante CC), que se define como inevitable. La educación es sin lugar a dudas una institución fundamental para la socialización en valores, actitudes y comportamientos pro-ambientales, pues serán las generaciones más jóvenes las que en mayor medida sufran y tengan que ges-tionar esos impactos.

¿Está el sistema educativo preparado para ello? Aquí presentamos un avance de la situación de la Comunidad Autónoma Extremeña, un trabajo planteado como investigación exploratoria, centrada en los niveles de la ESO. La Metodología utilizada tiene un carácter eminentemente cualitativo e incluye: una selección de colegios públicos y privados que no pretende ser representativa, entrevista a jefes de estudios y responsables del proyecto Eco-centros de dichos colegios, y un análisis documental de las leyes educativas nacionales y extremeñas y de los proyectos pedagógicos de los colegios seleccionados. Con la cautela que exige una investigación de estas características, los primeros avances no son muy optimistas, no perci-biéndose una política educativa concreta que tenga en cuenta esta cuestión.

\section{Palabras clave}

Cambio Climático, sistema educativo, ESO, currículo, Extremadura.

\section{Abstract}

Since the adoption by the UN General Assembly in 1988 of the Intergovernmental Panel On Climate Change (IPCC) have been five reports, the last one published late 2014, alerting us to the catastrophic consequences that the rising temperature of the planet will have for human societies and ecosystems that maintain life on earth. The report recommends that policy mak-ers take steps to facilitate citizens' resilience and adaptation to the global environmental change defined as inevitable.

Education is without a doubt a fundamental institution for socialization in pro-environmental values, attitudes and behaviours, especially of younger generations who will be the ones who suffer the most and have to manage those impacts. However, is the educational system pre-pared for it? Here we present an advance of the situation of the Autonomous Community of Extremadura. The work we propose as an exploratory research, centred on the levels of ESO. The Methodology used has a qualitative character and includes a selection of public and pri-vate schools that do not pretend to be representative, interviews with the head of studies of these schools, and a documentary analysis of the extreme educational laws and pedagogical projects of the selected schools.

With the caution required to be in a very early stage of research, the first advances are not very optimistic, not perceiving a concrete educational policy that considers that issue.

\section{Keywords}

Climate change, educational system, ESO, curriculum, Extremadura. 


\section{Introducción}

Las primeras voces de alarma sobre la degradación ambiental se oyeron a lo largo de la década de los 50 y 60 del pasado siglo (Riesman, 1950, Packard, 1961; Carson, 1962; Bookchin, 1962; Ehrlich, 1968, 1993; Commoner, 1972; Informe Meadows, 1972). Un posicionamiento crítico con el modelo de desarrollo económico que sustentaba el modo de vida occidental que, junto a un creciente cúmulo de evidencias sobre sus efectos más nocivos, condujeron a la celebración de la primera Conferencia Internacional sobre Medio Ambiente Humano (Estocolmo, 1972). Desde entonces los problemas ambientales no han hecho sino acrecentarse en todo el mundo, pero también la toma de conciencia sobre los mismos. Una buena prueba de la preocupación que la cuestión ambiental genera, son las sucesivas conferencias de Desarrollo Sostenible realizadas por la ONU y la firma de un extenso conjunto de tratados internacionales asumidos por la mayoría de los países miembros.

Uno de estos tratados es la Convención Marco de las Naciones Unidas sobre el Cambio Climático (CMNUCC) que más tarde llevaría al Protocolo de Kyoto (1998). La COP (Conferencia de las Partes) es el «órgano supremo» de la Convención, es decir su máxima autoridad con capacidad de decisión, y tiene como objetivo examinar la aplicación de la Convención y evaluar los efectos de las medidas adoptadas por las Partes. Desde su creación en 1995 se han realizado XXII Conferencias Internacionales sobre Cambio Climático (CC), la última en Marrakech (2016).

La estructura internacional de lucha contra el CC se complementa con el Panel Internacional de Expertos sobre Cambio Climático (IPCC, siglas en inglés), formado por centenares de científicos y expertos. Una de sus principales actividades es realizar una evaluación periódica de los conocimientos sobre el CC y elaborar informes-resúmenes para los responsables políticos, que deberían ser tenidos en cuenta en el diseño de políticas públicas. El último informe de evaluación, publicado en 2014, incide en la urgencia de la mitigación (reducción de las emisiones) y en la adaptación y resiliencia de las poblaciones a los impactos y escenarios de riesgo. Y una de las fórmulas propuestas para ello son las políticas educativas y su orientación hacia la sensibilización e integración de estas cuestiones; tanto en los sistemas educativos formales como informales.

A partir de estas evidencias, las principales agencias internacionales apuestan por la Educación como una herramienta fundamental para preparar a las generaciones actuales, pero sobre todo a las futuras, para enfrentar los efectos del CC. En 2009 (Bonn) la Conferencia Mundial de la UNESCO sobre Educación para un Desarrollo Sostenible abundó en esta idea, y muchos países han empezado a desarrollar estrategias. El Grupo de Trabajo sobre Objetivos, Audiencias y Estrategias en la Educación sobre el CC, promovido por el Consejo Nacional de Investigación de los Estados Unidos, advirtió en (Forrest, Feder, 2011) de cómo su país, a pesar de existir amplia información disponible, se enfrentaba a un vacío de conocimiento entre el público, tanto sobre los efectos, como sobre los desafíos y, también, oportunidades. Por ello planteaba la necesidad de mejorar tanto la educación en el campo de las Ciencias de la Tierra, como sobre todo sobre las dimensiones humanas y sociales del CC. Por su parte Muttarak and Lutz (2014) revisan la literatura sobre el tema y concluyen que la educación debería ser considerada un factor clave para reducir la vulnerabilidad a los desastres, y potenciar la capacidad adaptativa al cambio climático

En el caso de España no se ha diseñado una estrategia en esta dirección. Incluso el propio análisis sobre el conocimiento del problema por parte de la población ha quedado fuera de la agenda del Go- 
bierno, no involucrándose el Centro de Investigaciones Sociológicas en análisis en esta dirección ${ }^{2}$, si bien han aparecido informes financiados por fundaciones empresariales (Meira et al., 2009). Y aunque en 2004 desde el Centro Nacional de Educación Ambiental y la Oficina Española de CC se promovió un Grupo de Trabajo sobre Comunicación, Educación y Participación frente al CC, que ha realizado hasta 2016 trece seminarios monográficos ${ }^{3}$, sus implicaciones en el sistema educativo han sido escasas, más allá de los programas específicos de Educación Ambiental que distintos organismos públicos y privados desarrollan. Con un enfoque más amplio que incluye la información y la comunicación sobre CC, los trabajos del grupo de investigación RESCLIMA ${ }^{4}$ parten del presupuesto de que la alfabetización climática es una condición insuficiente para cambiar las actitudes y comportamiento de los escolares en relación al CC y se centran en analizar el factor social en la interacción clima-sociedad. En sus tesis defienden que la principal fuente de incertidumbre no está en las ciencias del clima, sino en las lecturas sociales, económicas y políticas que condicionan las respuestas dadas a la amenaza del CC. En uno de sus últimos trabajos analizan la presencia del CC en los libros de texto de secundaria, evidenciando la existencia, «de un marco reduccionista y convencional, centrado exclusivamente en la dimensión ambiental pero olvidando introducir la dimensión social» (Serantes, A. y Meira, P. 2016: 164),

Es evidente que en el caso de España es complicado plantear una estrategia educativa sobre un tema concreto, dado que las competencias en materia de Educación están transferidas a las Comunidades Autónomas. De ahí que sea necesario descender al nivel autonómico si quere-mos averiguar, atendiendo al objetivo de nuestra investigación, hasta qué punto el problema del CC se ha integrado en el currículo, o al menos lo ha percolado directa o indirectamente.

\section{Metodología}

El diseño metodológico, que no trata de ser representativo, se ha planteado como una investigación exploratoria. La estrategia de investigación utilizada ha sido la triangulación cualitativa que incluye, además de la consiguiente revisión bibliográfica previa, un análisis documental de las leyes educativas nacionales y las de la Comunidad Autónoma de Extremadura y la realización de nueve entrevistas en profundidad, presenciales y semi-estructuradas a jefes de estudios de los Institutos de Enseñanza Secundaria (IES) y a coordinadores del proyecto de Eco-centros. Las entrevistas se han realizado durante los meses de enero a mayo de 2017.

Otro elemento analítico de interés para nuestro estudio eran los proyectos educativos de los centros seleccionados, a los que finalmente hemos tenido que renunciar debido a las grandes dificultades para acceder a ellos. A pesar de ser documentos públicos no están accesibles en la web y ante nuestras peticiones para obtenerlos las respuestas siempre han sido evasivas.

En la selección de los centros educativos se han utilizado criterios de titularidad (pública/privada/concertada) socioeconómicos (rentas altas, medias y bajas del alumnado), y territoriales (zonas rurales y urbanas), con la intencionalidad de generar la máxima diversidad dentro de los escasos medios disponibles.

De los niveles académicos optamos por la educación secundaria por ser una etapa de consolidación de conocimientos impartidos en primaria, y de preparación para el Bachiller. Además el alumnado está en la fase vital en la que la familia ha dejado de ser el principal agente socializador, interviniendo en la

2 No obstante, tras serle presentada una propuesta (no adjudicada) de Panel de Preguntas sobre Cambio Climático, el CIS incorporó en uno de sus barómetros de 2016 algunas de las preguntas propuestas (Baigorri, Caballero, 2018).

3 Puede accederse al material generado en los seminarios en la web del Ministerio de Agri-cultura, Pesca, Alimentación y Medio Ambiente: https://goo.gl/jMXYZJ

4 Para más información véase http://www.resclima.info. 
construcción de su personalidad otros agentes externos como el grupo de iguales, o los medios de comunicación que influyen poderosamente en la adquisición de valores y actitudes; pero finalmente es sobre los conocimientos y valores transmitidos en el sistema educativo sobre los que los jóvenes racionalizan en ese periodo sus creencias y valores.

\section{Datos y resultados}

\section{1. Legislación educativa y descentralización}

La distribución de competencias educativas está regulada por la Ley Orgánica de Mejora de la Calidad Educativa (LOMCE), que establece en el art 6 bis una distribución de competencias entre el Estado, las Comunidades Autónomas y los Centros. Distingue tres grupos de asignaturas: troncales (T), específicas (E) y de libre configuración autonómica (LCA). A las Comunidades Autónomas se las faculta para completar el contenido de las troncales y establecer los contenidos tanto de las específicas como las de libre configuración (art. 6 bis, 2, c). Respecto a los Centros, se dice que podrán completar los contenidos de los bloques de asignaturas troncales, específicas y de LCA (art 6 bis, 2, c).

Este doble plano de descentralización educativa - esto es, la posibilidad de que gobiernos autonómicos y centros educativos puedan introducir cambios en el currículo- facilita, al menos desde el punto de vista normativo, una cierta diferenciación entre ellos, que se expresaría en distintas «identidades» pedagógicas entre unos y otros. En cierto sentido podemos decir que el regulador favorece la diversidad y con ello aumenta las posibilidades que tienen los padres a la hora de elegir para sus hijos el producto educativo, es decir, el centro, más afín a sus valores.

En este contexto descentralizador, la Ley 4/2011 de Educación de Extremadura es fruto del consenso político entre el gobierno de la Comunidad en aquel momento (PSOE) y el entonces principal partido de la oposición (PP). Fundamentada en las posibilidades que otorgaba la LOE, el artículo 141 desarrolla los pormenores de la autonomía pedagógica. Y atendiendo al mandato de la propia Ley de Educación de Extremadura, mediante el Decreto 104/2013, de 18 de junio, se regula el funcionamiento de las Redes de Apoyo Social e Innovación Educativa de la Comunidad Autónoma de Extremadura y se crea la modalidad de Red de Escuelas de Investigación, Desarrollo Tecnológico e Innovación Educativa. Una de estas redes es la Red de Eco-centros de la que más adelante nos ocuparemos.

\subsection{El tratamiento de Cambio Climático en la ESO}

En este apartado vamos a comprobar las diferencias que existen, a la hora de trabajar el término de CC, entre unas y otras asignaturas Para ello utilizamos un indicador básico, el número de veces que el término CC aparece en los contenidos, criterios de evaluación y estándares de aprendizaje del currículo de Secundaria. También tendremos en cuenta en qué asignatura aparece el término, y si la asignatura es troncal, específica o de LCA, lo que a su vez nos informa de la importancia asignada.

La ley educativa extremeña recoge la obligatoriedad de las asignaturas troncales y específicas establecidas en las leyes educativas vigentes (tabla $I)^{5}$ que se imparten en todo el territorio nacional. Las primeras suponen como mínimo un 50\% del horario lectivo, el otro (como máximo) 50\% se reparte entre las asignaturas específicas y las de LCA. Pues bien, el término de CC solamente aparece en las siguientes asignaturas específicas:

\footnotetext{
5 http://www.mecd.gob.es/dms/mecd/educacion-mecd/mc/lomce/lomce/paso-a-paso/LOMCEd_pasoapaso_secundaria-v4/LOMCEd_pasoapaso_secundaria $\% 20 \mathrm{v} 4 . \mathrm{pdf}$
} 
Valores Éticos. Asignatura de primer ciclo de la ESO, aparece como estándar de aprendizaje en el Bloque 6, denominado «Los valores éticos y su relación con la ciencia y la tecnología».

4.3. Selecciona y contrasta información, en colaboración grupal, acerca de algunas de las amenazas que, para el medio ambiente y la vida, está teniendo la aplicación indiscriminada de la ciencia y la tecnología, tales como: la explotación descontrolada de los recursos naturales, la destrucción de hábitats, la contaminación química e industrial, la lluvia ácida, el CC, la desertificación, etc. (BOE n. ${ }^{\circ}$, pág. 541).

Ciencias Aplicadas a la Actividad Profesional. Asignatura de 4. ${ }^{\circ}$ de la ESO, aparece una sola vez como criterio de evaluación en el bloque 2 denominado Aplicaciones de la ciencia en la conservación del medio ambiente: «2. Contrastar en qué consisten los distintos efectos medioambientales tales como la lluvia ácida, el efecto invernadero, la destrucción de la capa de ozono y el CC». (BOE n³, pág. 225).

Cultura Científica. Asignatura de $4 .^{\circ}$ de la ESO. El término aparece dos veces una en la introducción a la asignatura: «En la vida diaria estamos en continuo contacto con situaciones que nos afectan directamente, como las enfermedades, la manipulación y producción de ali-mentos o el CC, situaciones que los ciudadanos del siglo XXI debemos ser capaces de entender» (BOE, después de n. ${ }^{\circ}$ 3, pág. 465). Y otra como estándar de aprendizaje evaluable: «2.1. Reconoce los efectos del CC, estableciendo sus causas» (DOE n. ${ }^{\circ}$ 3, pág. 466).

En el resto de asignaturas troncales del currículo de la ESO no aparece el término CC, ni como contenido, ni como criterio de evaluación, ni como estándar de aprendizaje evaluable. Este es el grado de importancia que se le concede al concepto de CC en asignaturas troncales obligatorias establecidas a nivel central y por tanto presentes en el currículo de todas las CC. AA. lo que demuestra el nulo interés que para las autoridades educativas tiene este asunto.

\section{Tabla I. Mapa de asignaturas de la ESO}

PRIMER CICLO $10^{\circ},{ }^{\circ} .^{\circ}$ Y.$^{\circ}$ DE LA ESO

\begin{tabular}{|c|c|c|c|}
\hline & PRIMERO ESO & SEGUNDO ESO & TERCERO ESO \\
\hline Asignaturas Troncales & Biología y Geología & Física y Química & Biología y Geología \\
\hline \multirow{7}{*}{$\begin{array}{l}\text { El horario lectivo de estas materias ocupará como } \\
\text { mínimo, el } 50 \% \text { del total del horario }\end{array}$} & Geografía e Historia & Geografía e Historia & Física y Química \\
\hline & Lengua Castellana y Litera-tura & Lengua Castellana y litera-tura & Geografía e Historia \\
\hline & Matemáticas & Matemáticas & Lengua Castellana y Literatura \\
\hline & Primera Lengua Extranjera & Primera Lengua Extranjera & Primera Lengua Extranjera \\
\hline & & & Materias de opción \\
\hline & & & $\begin{array}{l}\text { Matemáticas Orientadas a la Enseñanzas } \\
\text { Académicas }\end{array}$ \\
\hline & & & $\begin{array}{l}\text { Matemáticas Orientadas a la Enseñanzas } \\
\text { Aplicadas }\end{array}$ \\
\hline Asignaturas Específicas & \multicolumn{3}{|c|}{ Educación Física; Religión ${ }^{\star}$ y Valores Éticos ${ }^{\star}$} \\
\hline \multirow[t]{2}{*}{ En cada uno de los cursos } & \multicolumn{3}{|c|}{ Un mínimo de una y un máximo de cuatro de las siguientes asignaturas que podrán ser diferentes en cada uno de los cursos: } \\
\hline & \multicolumn{3}{|c|}{$\begin{array}{l}\text { Cultura Clásica, Iniciación a la Actividad Emprendedora y Empresarial, Música, Tecnología, Educación Plástica, Visual y Audiovisual, Segunda Lengua } \\
\text { Extranjera, Religión }{ }^{\star \star} \text { y Valores Éticos** }\end{array}$} \\
\hline \multirow[t]{2}{*}{ Asignaturas de L.C.A. } & \multicolumn{3}{|c|}{ Lengua Cooficial y Literatura (recibirá un tratamiento análogo al de la materia de Lengua Castellana y Literatura) } \\
\hline & \multicolumn{3}{|c|}{ Asignaturas espećificas no cursadas o materias por determinar } \\
\hline
\end{tabular}


SEGUNDO CICLO, $4^{\circ}$ DE LA ESO

\begin{tabular}{|c|c|c|}
\hline \multirow{12}{*}{$\begin{array}{l}\text { El horario lectivo de estas materias ocupará como } \\
\text { míni-mo, el } 50 \% \text { del total del horario }\end{array}$} & $\begin{array}{l}\text { Enseñanzas Académicas } \\
\text { (hacia Bachillerato) }\end{array}$ & $\begin{array}{l}\text { Enseñanzas Aplicadas } \\
\text { (hacia la Formación Profesional) }\end{array}$ \\
\hline & \multirow{4}{*}{\multicolumn{2}{|c|}{$\begin{array}{l}\text { Troncales Generales } \\
\text { Geografía e Historia } \\
\text { Lengua Castellana y Literatura } \\
\text { Primera Lengua Extranjera }\end{array}$}} \\
\hline & & \\
\hline & & \\
\hline & & \\
\hline & Matemáticas orientadas a las enseñanzas académicas & Matemáticas orientadas a las enseñanzas aplicadas \\
\hline & \multicolumn{2}{|c|}{ Troncales de cada opción } \\
\hline & A elegir dos entre las siguientes: & A elegir dos entre las siguientes: \\
\hline & Biología y Geología & Ciencias aplicadas a la actividad profesional \\
\hline & Economía & Iniciación a la actividad emprendedora y empresarial \\
\hline & Física y Química & Tecnología \\
\hline & \multicolumn{2}{|l|}{ Latín } \\
\hline \multirow{3}{*}{$\begin{array}{l}\text { Asignaturas Específicas } \\
\text { en cada uno de los cursos }\end{array}$} & \multicolumn{2}{|l|}{ Educación Física; Religión ${ }^{\star}$ y Valores Éticos* } \\
\hline & \multicolumn{2}{|c|}{ Un mínimo de una y un máximo de cuatro de las siguientes asignaturas que podrán ser diferentes en cada uno de los cursos: } \\
\hline & \multicolumn{2}{|c|}{$\begin{array}{l}\text { Artes Escénicas y Danza, Cultura Cientifica, Cultura Clásica, Filosofía, Música, Tecnología de la Información y la Comunicación, Segunda Lengua Extranje- } \\
\text { ra, Educación Plástica, Visual y Audiovisual, Una materia del bloque de asignaturas troncales no cursadas, Religión }{ }^{\star \star} \text { y Valores Éticos** }\end{array}$} \\
\hline \multirow[t]{3}{*}{ Asignaturas de L.C.A. } & \multicolumn{2}{|c|}{ Lengua Cooficial y Literatura (recibirá un tratamiento análogo al de la materia de Lengua Castellana y Literatura) } \\
\hline & \multicolumn{2}{|l|}{ Asignaturas específicas no cursadas o materias por determinar } \\
\hline & \multicolumn{2}{|c|}{ Materias de ampliación de los contenidos de algunas de las materias de los bloques de asignaturas troncales o específicas } \\
\hline
\end{tabular}

* Se cursarán en todos los cursos

** Si los padres, madres o tutores legales, o en su caso los estudiantes, no la han escogido anteriormente.

En negrita, cursiva y subrayada las asignaturas dónde aparece el término CC (la señalización es nuestra).

Fuente: Ministerio de Educación, Cultura y Deporte. Elaboración propia.

Esta es pues la situación curricular común a todas las CC. AA. Para conocer las diferencias entre ellas, tenemos que acudir a las asignaturas de LCA. La Tabla II recoge estas asignaturas para la ESO en Extremadura, aprobadas mediante el Decreto 98/2016, de 5 de julio.

Tabla II. Asignaturas de Libre Configuración de la Comunidad de Extremadura

\begin{tabular}{|c|c|}
\hline CURSO & ASIGNATURA \\
\hline $1 .{ }^{0} \mathrm{ESO}$ & Segunda lengua Extranjera \\
\hline \multirow[t]{2}{*}{$2 .{ }^{\circ} \mathrm{ESO}$} & Segunda Lengua Extranjera \\
\hline & Educación para la Ciudadanía y Derechos Humanos \\
\hline \multirow[t]{3}{*}{ 3. ${ }^{0} \mathrm{ESO}$} & Segunda Lengua Extranjera \\
\hline & Cultura Clásica \\
\hline & Iniciación a la actividad emprendedora y empresarial \\
\hline
\end{tabular}

Fuente DOE. Elaboración propia.

El término CC aparece una sola vez como estándar de aprendizaje evaluable dentro de la asignatura Educación para la Ciudadanía y Derechos Humanos, en el bloque VI denominado La ciudadanía en el siglo XXI: «47.2 Evalúa que combatir el CC es un reto tanto individual como colectivo» (DOE n. ${ }^{\circ} 129$, pág. 17886).

Este estándar de aprendizaje está vinculado a uno de los criterios de evaluación que valora los contenidos sobre Ética, Ciencia y Tecnología

47. definir problemas medioambientales relacionados con la ciencia y la tecnología, así como en qué 
consiste el Protocolo de Kioto y la Cumbre del clima de París de 2015 Propone actitudes civicas relacionadas con el desarrollo sostenible y el respeto al medioambiente. (DOE n. ${ }^{\circ 129}$, pág. 17886).

A la vista de lo expuesto, es evidente que la administración educativa extremeña tampoco ha realizado grandes esfuerzos por incorporar contenidos sobre CC en la parte del currículo que puede regular, a pesar de presumírsele, al menos desde el punto de vista ideológico, una acti-tud favorable en la lucha contra el CC.

\subsection{La Red de Eco-centros}

La preocupación por la cuestión ambiental y su tratamiento desde la escuela ha estado presente en el ánimo de la Junta de Extremadura desde que asumió las competencias educativas, aunque con resultados muy dispares. Un ejemplo fue el programa Centros Verdes, durante los cursos 1993/94 y 1994/95, que a juicio de Conde M. C et al., (2003) no tuvieron la continuidad y el necesario apoyo que requerían.

A estos primeros proyectos de carácter puntual les siguieron otros de más envergadura y con mayor vocación de continuidad en el tiempo. Así, en 2003 se pone en marcha un proyecto de innovación educativa en educación ambiental denominado Eco-centros, que se diseña priorizando un modelo de participación abierto a la comunidad, con mecanismos democráticos de organización y funcionamiento. Se establecen las líneas generales de trabajo sobre energía, agua, residuos, transporte y entorno, e inicialmente se seleccionan 13 centros de infantil y primaria, uno por cada Centro de Profesores y Recursos (CPR) de la región.

El éxito del proyecto lleva a la Junta de Extremadura a extender el modelo a otras temáticas mediante del Decreto 104/2013, de 18 de junio, que regulaba el funcionamiento de la Red de Escuelas de Investigación, Desarrollo Tecnológico e Innovación Educativa.

Finalmente, y tras la experiencia de gestión y evolución de la situación de los centros educativos en estas redes de investigación e innovación, se introducen algunos cambios que quedan recogidos en un nuevo Decreto, el 75/2015, de 21 de abril, por el que se regula el funcionamiento de las Redes ${ }^{6}$ de Apoyo Social e Innovación Educativa en los centros educativos sostenidos con fondos públicos de enseñanza no universitaria de la Comunidad Autónoma de Extremadura. El Decreto se inspira en algunos de los principios esenciales de la Ley Orgánica 2/2006, de 3 de mayo, de Educación; en la Ley Orgánica 8/2013, de 9 de diciembre, para la mejora de la calidad educativa; y en el ámbito regional, en la Ley 4/2011, de 7 de marzo, de Educación de Extremadura.

En la actualidad existen siete redes con temáticas muy distintas pero con objetivos generales organización y funcionamiento muy similares. En este aspecto y sin ánimo de ser exhaustivos nos interesa destacar la vinculación de la filosofía del proyecto de Redes que impregna el proyecto educativo de centro y se concreta en el proyecto curricular, el carácter voluntario de participación de los centros en cualquiera de las redes, la posibilidad de adscribirse a más de una, y la exigencia de poner en marcha un programa por un periodo de tres años.

En el caso de los centros adscritos a la red de Eco-centros, además de respetar los objetivos generales establecidos en el artículo 3 del Decreto 104/2013, tienen como objetivos específicos (anexo I):

\footnotetext{
Red Extremeña de Eco-centros, Red Extremeña de Escuelas Promotoras de Salud, Red Extremeña de Escuelas por una Cultura de Paz, Igualdad y No Violencia Red Extremeña de Escuelas de Inteligencia Emocional, Red Extremeña de Centros Promotores de la Actividad Físico-Deportiva, Red Extremeña de Escuelas Emprendedoras y Red de Escuelas de Investigación Educativa
} 
$1{ }^{\circ}$ Hacer del centro educativo un proyecto susceptible de cambios y mejoras, tanto en el ámbi-to ecológico como social, coherente con los principios de la Educación Ambiental e impulsa-do por su propia comunidad educativa.

2.' Abordar en el Proyecto de Redes de centro los temas de trabajo que se indican a continua-ción: energía, agua, residuos, transporte y entorno. (DOE, núm. 121, pág. 14940).

La red de Eco-centros es la más numerosa de las redes, constituida por 25 centros educativos de los cuales 9 son IES. Según el Registro Estatal de Centros Docentes no Universitarios dependiente del Ministerio de Educación, Cultura y Deporte, en Extremadura existen un total de 113 centros de Educación Secundaria Obligatoria de carácter público. Por tanto, los IES que participan en la Red de Eco-centros representan el $8 \%$ del total y solamente en 2 de ellos existe continuidad en la pertenencia a la red entre los niveles primaria y secundaria. Estos datos ponen pues en evidencia al menos dos debilidades: primero, el escaso número de IES que participan en el proyecto de Eco-centros y por tanto la reducida exposición del alumnado a estos temas; y segundo, la falta de continuidad de esa participación entre unos niveles educativos y otros, evidenciando en suma una falta de coherencia pedagógica y también de previsión política.

\section{4. ¿Qué hay del Cambio Climático en la Red de Eco-centros?}

Según el discurso normalizado, una de las implicaciones más significativas de la pertenencia a este tipo de Redes es que los proyectos educativos de centro y los proyectos curriculares se impregnan de la filosofía de la red a la que pertenecen, y la expresan tanto en forma de identidad pedagógica como en concreción curricular. Por ello, en el caso de los centros que pertenecen a la red de Eco-centros cabría esperar una fuerte presencia de principios ecológicos en los proyectos educativos de centro y una clara estrategia transversal de «ambientalización» de los proyectos curriculares y de la vida del centro educativo en toda su extensión.

Con respecto a cómo se incardina la filosofía de los principios ambientalistas en el proyecto educativo de centro poco podemos decir porque no ha sido posible acceder a ninguno de ellos ${ }^{7}$. A pesar de ser un documento público, ninguno de los centros seleccionados lo tiene en abierto y cuando se pregunta expresamente por ello las respuestas son evasivas.

En cuanto a la transversalidad y concreción de la cuestión ambiental en los proyectos curriculares, tampoco las respuestas son claras; en general la mayor o menor vinculación con las asignaturas dependen, más que del proyecto curricular, de la sensibilización e implicación del profesorado con estos temas.

En las entrevistas mantenidas con los coordinadores del proyecto de Eco-centros de los centros seleccionados, se ha puesto de manifiesto que la implicación del gobierno regional fue efectiva desde el inicio del proyecto (curso 2003/04) hasta prácticamente el comienzo de la crisis económica en 2008, momento en que se «desinfla» y «se deja caer», desapareciendo gran parte de la dotación económica, la figura del coordinador regional, las actividades intercentros, etc.

Esta falta de continuidad y/o abandono del proyecto cuando los recursos son escasos, es definida por algunos informantes como de «lucimiento político» en el sentido de que «se actúa de cara a la ga-

\footnotetext{
Esa situación no está solo referida a los Eco-centros; en general de todos los centros seleccionados para realizar nuestra investigación solo aquellos de carácter privado/concertado y religioso tienen el acceso abierto a su pro-yecto educativo.
} 
lería, porque queda muy bien y luce mucho eso de los Eco-centros, pero luego a la hora de la verdad la implicación de la administración es bastante escasa». A nuestro juicio esta situación, que hace depender la existencia y éxito de un proyecto de estas características en función de los recursos disponibles, muestra el carácter periférico y ocasional que los temas ambientales tienen en la agenda educativa extremeña, mostrando la falta de una política educativa que aborde esta cuestión en profundidad.

En relación al CC las entrevistas evidencian que el concepto no se trabaja directamente, aunque sí de forma indirecta al tratar los temas de reciclaje, ahorro de agua o de energía, etc. En las entrevistas aparece de nuevo la sensibilidad y el interés del profesorado para vincular el CC con las actividades desarrolladas por el Eco-centro. El mayor nivel de conexión lo encontramos en los impactos que el CC tiene en los ecosistemas naturales. Es mínima la vinculación del CC con los factores sociales que lo originan y lo soportan, el impacto desigual de sus efectos en las sociedades actuales y futuras, o las respuestas políticas globales que se articulan para limitarlo (Protocolo de Kyoto, Acuerdo de París, etc.); tampoco se vinculan las actividades con los acuerdos y recomendaciones de los informes del IPCC. A nuestro juicio estas son algunas de las debilidades de los Eco-centros; es evidente que, en su programa, de carácter fuertemente biologista, faltan las dimensiones sociales y políticas de la interacción clima/ sociedad y más ampliamente medioambiente y sociedad. La ausencia de estas dimensiones dificulta en los estudiantes una visión global del problema al que nos enfrentamos y retra-san la adopción de las respuestas más adecuadas para mitigarlo.

La escasa presencia de contenidos ambientalistas en el currículo y el carácter voluntario de la participación tanto del profesorado como del alumnado en el Ecocentro, genera una brecha ambiental importante ampliando las diferencias entre quienes están expuestos a los efectos del proyecto y aquellos que no participan. Esta voluntariedad dificulta la consolidación general de actitudes pro-ambientales dentro del centro, que puedan extenderse al resto de la comunidad. En este sentido uno de nuestros informantes al relatar su experiencia como profesor de Física y Química y coordinador del Eco-centro dice:

(...) Los alumnos se implican poco, y los profesores también. Los alumnos distinguen entre lo que hacen en clase y fuera. Por ejemplo el aborro del agua, saben cómo ahorrar agua pero en cuanto salen de clase lo olvidan. Aprenden cosas para aprobar pero en su vida estas cosas están al margen.

\section{Conclusiones}

Con la cautela con la que deben tomarse las conclusiones en un estudio exploratorio, podemos avanzar algunas conclusiones provisionales que serán necesario confirmar y profundizar en un estudio descriptivo más amplio. En este caso se ha basado, según se ha señalado, en un modesto trabajo de campo, sin grandes ambiciones dada la inexistencia de medios, pero nos ha permitido conformar las bases metodológicas para una investigación de más enjundia sobre el tema.

La principal evidencia, por ahora, es la escasa presencia del término CC en el currículo de la ESO, tanto en las asignaturas troncales como en las específicas y de LCA. Si la escuela expresa y reproduce los valores dominantes de una sociedad, resulta evidente que los valores ambientales no forman parte del ideario colectivo de la sociedad española, y en particular de la sociedad extremeña, a pesar de que en este caso «lo ambiental» forma parte del relato identitario regional si atendemos a los discursos políticos, promoción exterior, etc. 
Entendemos que existe desde todos los ámbitos de la administración educativa un claro desafecto, por otro lado incomprensible, con el reto que para nuestras sociedades supone el CC. Y cuando de las instituciones educativas se espera que eduquen a los ciudadanos del futuro en el presente, resulta intolerable la falta previsión que se evidencia.

En el caso de la administración educativa extremeña encontramos ecos de una cierta sensibilidad ambiental acorde a sus postulados ideológicos. Pero su concreción en experiencias coyunturales e incompletas, como es el proyecto de Eco-centros, demuestra el carácter periférico que en realidad la cuestión ambiental tiene en su política educativa. Queda lejos de la «climate literacy» (US Global Change Research Program, 2009) promovida para la Educación Secundaria por el gobierno Obama, hoy desmantelada por la administración Trump.

Entre los resultados esperados adicionales de nuestra investigación ha de estar el informe dirigido a los responsables educativos, haciéndoles ver la contradicción que supone poner en marcha un modelo basado en el eslogan de la de Economía Circular y no incorporar la alfabetización climática en los currículos de la ESO.

Y entre las líneas futuras de investigación nos planteamos en contrastar el sistema educativo español con contrastar con otros sistemas educativos. Ya lo estamos investigando para el caso de Portugal, que tiene un modelo educativo más centralizado, y de lograr financiación nos plantearíamos hacer el contraste con países supuestamente más avanzados educativamente, como Finlandia. Pero a nivel local también creemos que hay un campo importante de análisis pendiente, desde la Sociología de la Educación, sobre la propia preparación de los profesores para afrontar estas cuestiones, que a menudo se confunden con la Educación Ambiental, pero que son (a pesar de su cercanía) de otra naturaleza.

\section{Referencias bibliográficas}

Arto, Mónica, Meira, Pablo et al., (Coords.) (2017). RESCLIMA: Aproximación ás claves sociais e educativas do cambio climático. Ferrol: Aldine Editorial.

Baigorri, Artemio y Caballero, Manuela (2018): "Negacionismo, políticas demoscópicas y currículum de fracasos. El caso del cambio climático en España”. Aposta. Revista de Ciencias Sociales, 77, 8-58.

Bookchin, Murray (1962). Our Synthetic Environment. New York: Knopf. Recuperado de: http://dwardmac.pitzer.edu/Anarchist_Archives/bookchin/syntheticenviron/osetoc.html.

Carson, Rachel (1962). Silent Pring. Boston: Houghton Mifflin.

Conde, $\mathrm{M}^{\mathrm{a}}$ del Carmen et al., (Coord.) (2003). ECO-CENTROS. Una experiencia de innovación educativa en Educación Ambiental. Mérida: Junta de Extremadura.

Commoner, Barry (1971). The Closing circle. Nueva York: Knopf (edición española El círculo que se cierra. Barcelona: Plaza y Janés, 1978).

Decreto 104/2013, de 18 de junio, por el que se regula el funcionamiento de las Redes de Apoyo Social e Innovación Educativa de la Comunidad Autónoma de Extremadura y se crea la modalidad de Red de Escuelas de Investigación, Desarrollo Tecnológico e Innovación Educativa. Diario Oficial de Extremadura. 21 de junio 2013, núm. 121, pp. 14920-14947 
Decreto 98/2016, de 5 de julio por el que se establecen la ordenación y el currículo de la Educación Secundaria Obligatoria y del Bachillerato para la Comunidad Autónoma de Extremadura. Diario Oficial de Extremadura, 6 de julio de 2016, núm. 129, pp. 17347-18550

Decreto, el 75/2015, de 21 de abril, por el que se regula el funcionamiento de las Redes de Apoyo Social e Innovación Educativa en los centros educativos sostenidos con fondos públicos de enseñanza no universitaria de la Comunidad Autónoma de Extremadura. Diario Ofi-cial de Extremadura. 27 de abril 2015, núm. 79, pp.13557-13592

Meadows, Donella H. et al., (1972). Los Limites del crecimiento: informe al Club de Roma sobre el predicamento de la humanidad. México D.F. Fondo de Cultura Económica.

Ehrlich, Paul (1968). The population bom. New York: Ballantines Boocks.

Ehrlich, Paul y Ehrlich, Anne (1993). La explosión demográfica. Elprincipal problema ecológico. Barcelona: Salvat.

Forrest, Sherrie; Feder, Michael (Ed.) (2011). Climate Change Education. Goals. Audiences, and Strrategies. Washington: The National Academies Press.

Heiss, Julia (Coord.) (2010). Decade of Education for Sustainable Developement. Paris: UNESCO.

Ley Orgánica 8/2013, de 9 de diciembre, para la mejora de la calidad educativa. Boletín Oficial del Estado. Madrid. 10 de diciembre de 2013, núm. 295, pp. 97858 a 97921.

Ley 4/2011 de Educación de Extremadura. Diario Oficial de Extremadura. 9 marzo 2011, núm. 47, pp. $5952-6035$.

Meira, Pablo; Arto, Mónica y Montero, Pablo (2009). La sociedad ante el Cambio Climático. Conocimietos, valoraciones y comportamientos en la población española. Fundación Mapfre.

National Research Council. (2011): “Climate Change Education: Goals, Audiences, and Strategies. A Workshop Summary" in Sherrie Forrest and Michael Feder (Rapporteurs): Board on Science Education, Division of Behavioral and Social Sciences and Education. Washington, DC: The National Academies Press.

Packard, Vance (1961). Los artifices del derroche. Buenos Aires: Editorial Sudamericana.

Riesman, David (1965). Abundancia ¿̇para qué? México D.F.: Fondo de Cultura Económica.

Serantes-Pazos, Araceli; Meira, Pablo (2016): "El cambio climático en los libros de texto de la Educación Secundaria Obligatoria o una crónica de las voces ausentes”. Documentación Social, 138, 153-170.

US Global Change Research Program (2009). Climate Literacy: The Essential Principles of climate Science.

\section{Webgrafía}

www.boe.es

www.mecd.gob.es

https://www.educarex.es/

www.resclima.info

https://www.climate.gov/teaching/essential-principles-climate-literacy/essential-principles-climate-literacy https://goo.gl/jMXYZJ 


\section{Notas biográficas}

Manuela Caballero Guisado es Diplomada en Relaciones Laborales, Licenciada en Ciencias Políticas y Sociología, Doctora en Sociología y miembro del grupo de investigación Análisis de la Realidad Social de la Universidad de Extremadura, de la que es profesora desde 2009. Antes trabajó durante casi dos décadas como agente de desarrollo local. Desde 2015 lleva la secretaría del Comité 21 (Medio Ambiente y Sociedad) de la Federación Española de Sociología.

Artemio Baigorri Agoiz es licenciado en Ciencias Políticas y Sociología (especialidad de Psicología Social) y Doctor en Sociología. También cursó estudios de Ciencias de la Información. Ejerció el periodismo en los años 70, y la consultoría urbano-territorial en los 80, del siglo XX. Desde 1985 reside por elección en Badajoz, Extremadura, y desde 2001 es profesor titular de su universidad, en la que imparte desde 1996. Ha dirigido medio centenar de proyectos y convenios de investigación pura y aplicada, publicado más de un centenar de libros, capítulos de libro y artículos como autor o coautor, y dirigido seis tesis doctorales. 